\title{
Critical velocity, vortex shedding and drag in a unitary Fermi superfluid
}

\author{
F. Ancilotto ${ }^{1,2}$, L. Salasnich ${ }^{1}$, and F. Toigo ${ }^{1,2}$ \\ ${ }^{1}$ Dipartimento di Fisica e Astronomia "Galileo Galilei" and CNISM, \\ Università di Padova, Via Marzolo 8, 35122 Padova, Italy \\ ${ }^{2}$ CNR-IOM Democritos, via Bonomea, 265 - 34136 Trieste, Italy
}

\begin{abstract}
We study the real-time motion of a microscopic object in a cold Fermi gas at unitary conditions by using an extended Thomas-Fermi density functional approach. We find that spontaneous creation of singly quantized vortex-antivortex pairs occurs as a critical velocity is exceeded, which leads to a drag between the moving object and the Fermi gas. The resulting force is linear in the velocity for subsonic motion and becomes quadratic for supersonic motion.

PACS numbers: 05.30.Fk, 03.75.Ss, 67.85.-d
\end{abstract}

\section{INTRODUCTION}

A Fermi gas of atoms at unitary conditions is characterized by a divergent s-wave scattering length [1], which makes it a unique system, being at the same time dilute and strongly interacting. In this regime all scales associated with interactions disappear from the problem and the energy of the system is expected to be proportional to that of a non interacting fermions system. The unitary Fermi gas (UFG) at low energies is known to be superfluid, and the most striking manifestation of its phase coherence has been the experimental observation of vortices in a rotating UFG of ${ }^{6} \mathrm{Li}$ atoms [2].

Vortex dipoles in a Fermi gas were studied theoretically, within an extended Thomas-Fermi approach, in the BCS limit [3]. Vortex solutions within the same approach have been studied in Ref. [4], where the rotation of the Fermi system was predicted to generate a giant vortex in the presence of strong anharmonicity in the confining potential. Recently [5] a time-dependent Bogoliubov deGennes (BdG) approach has been used to study the 3-D, real-time formation of vortices in a UFG. Surprisingly, one of the main conclusion in Ref. [5] is that the system remains superfluid even when stirred at supercritical speed. Single vortex solutions in the Ginzburg-Landau regime of a trapped superfluid Fermi gase have been studied in Ref. [6] and Ref. [7]. In a dilute Fermionic superfluid a vortex state is characterized by a strong density depletion along the vortex core. The depletion is however not complete, according to the BdG calculations of Refs. [8, 9].

Recently it has been remarked [10] that the superfluid unitary Fermi gas can be efficiently described at zero temperature by phenomenological density functional theory. Density functionals of different flavours have been proposed by different theoretical groups. Bulgac and $\mathrm{Yu}$ have introduced a superfluid density functional based on a Bogoliubov-de Gennes approach to superfluid fermions [11, 12]. Papenbrock and Bhattacharyya [13] have instead proposed a Kohn-Sham density functional with an effective mass to take into account nonlocality effects. Here we adopt instead the extended Thomas-Fermi functional of the UFG that we have proposed few years ago
[14] and which has been used recently to successfully address a number of properties of such system [14 18].

Our extended Thomas-Fermi (ETF) density functional approach for the UFG [14, 15] is based on the following extended hydrodynamics equations:

$$
\begin{aligned}
\frac{\partial n}{\partial t}+\boldsymbol{\nabla} \cdot(n \mathbf{v}) & =0, \\
m \frac{\partial \mathbf{v}}{\partial t}+\boldsymbol{\nabla}\left[\frac{m}{2} v^{2}+U(\mathbf{r}, \mathbf{t})+\xi \frac{\hbar^{2}}{2 m}\left(3 \pi^{2}\right)^{2 / 3} n^{2 / 3}\right. & \\
\left.-\lambda \frac{\hbar^{2}}{2 m} \frac{\left(\nabla^{2} \sqrt{n}\right)}{\sqrt{n}}\right] & =\mathbf{0}
\end{aligned}
$$

where $n(\mathbf{r}, t)$ is the time-dependent scalar density field and $\mathbf{v}(\mathbf{r}, t)$ the time-dependent velocity field. Here $U(\mathbf{r}, \mathbf{t})$ is the external potential and $\xi=0.40$ and $\lambda=1 / 4$ [15, 18]. The above equations describe accurately various static and dynamical properties of the UFG. The term multiplied by $\lambda$ is found to be crucial to accurately describe surface effects, in particular in systems with a small number of atoms, where the Thomas-Fermi (local density) approximation fails [14]. We have shown that when fast dynamical processes occur and/or when shock waves come into play such term is necessary also in the large $\mathrm{N}$ limit [18], where results in quantitative agreement with experiments of real-time collision of strongly interacting Fermi gas clouds at unitarity [19] can be obtained [18.

We use this method here to study the motion of a microscopic 2-dimensional object with circular shape in a UFG and the associated process of vortex shedding, once the object velocity exceeds a critical value. Experimental realization of this geometry would imply, for instance, moving a far-detuned laser beam through a trapped condensate.

\section{CRITICAL VELOCITY AND DRAG IN THE UNITARY FERMI GAS}

For a dilute Fermi gas the long wavelength elementary excitations are sound waves, and the Landau criterion for the critical velocity $v_{c}=(\epsilon / p)_{\min }$ for breakdown of superfluidity gives $v_{c}=c_{1}$. However, for fluid flowing 
past an object (or an atomic impurity as well), the local velocity near the object surface can become supersonic even when the fluid velocity far from the object, $v_{\infty}$, is subsonic (the maximum velocity of, e.g., a 2-dimensional flow of an incompressible fluid past a circular obstacle is reached on the perimeter of the obstacle, where it is $\left.\sim 2 v_{\infty}\right)$.

An estimate for the critical velocity for a UFG can be obtained as follows. At stationary conditions Eq. (2) provides the Bernoulli equation for the UFG: (in the following $\left.\alpha \equiv \xi \frac{\hbar^{2}}{2 m}\left(3 \pi^{2}\right)^{2 / 3}\right)$

$$
-\lambda \frac{\hbar^{2}}{2 m} \frac{\left(\nabla^{2} \sqrt{n}\right)}{\sqrt{n}}+\alpha n^{2 / 3}+U(\mathbf{r})+\frac{m}{2} v^{2}=\text { const }
$$

By assuming that the quantum term in the previous equation is negligible in the spirit of the long-wavelength limit approximation, and calling $n_{0}$ the (uniform) density far from an impenetrable object, where $v \sim v_{\infty}$, one finds:

$$
n(\mathbf{r})=\left[n_{0}^{2 / 3}+\frac{m}{2 \alpha}\left(v_{\infty}^{2}-v^{2}\right)\right]^{3 / 2}
$$

outside the object and $n=0$ within it (the interaction $\mathrm{U}$ between the object and the fluid only provides the excluded volume condition).

The above equation may be written in terms of the local

$$
c_{l o c} \equiv \sqrt{\frac{\xi}{3}} v_{F}(\mathbf{r})=\sqrt{2 \alpha n(\mathbf{r})^{2 / 3} /(3 m)}
$$

and bulk

$$
c_{1} \equiv \sqrt{\frac{\xi}{3}} v_{F, \infty}=\sqrt{2 \alpha n_{0}^{2 / 3} /(3 m)}
$$

speeds of sound as:

$$
c_{l o c}^{2}=c_{1}^{2}+\left(v_{\infty}^{2}-v^{2}\right) / 3
$$

When $v \sim c_{l o c}$ local instabilities develop, leading to the release of vortices [20]. The maximum local velocity of a fluid flowing past an impenetrable cylindrical object, normally to its axis, is $v \sim 2 v_{\infty}$ (on the surface of the cylinder and tangent to it). Using this value in (7) one can solve the equation for $v_{\infty}$, thus providing an approximate value for the critical velocity of the fluid flowing past a stationary object (or, equivalently, of a moving object in the fluid at rest):

$$
v_{c}=c_{1} / \sqrt{5}
$$

This value is very similar to the one, $v_{c} \sim \sqrt{2 / 11} c_{1}$ obtained for the BEC case, using a similar argument, in Ref. [20]. For a spherical object the equatorial velocity is instead $v \sim 3 v_{\infty} / 2$, giving for the critical velocity of a sphere moving in a UFG $v_{c}=\sqrt{3 / 8} c_{1}$.
By combining the two equations (1) and (2) one can derive the equation for the momentum conservation (summation over repeated indices is implied):

$$
\partial_{t} J_{k}+\partial_{i} T_{i k}+\rho \partial_{k}(U / m)=0
$$

where $J_{k}=\rho v_{k}$ is the supercurrent density and the stress tensor $T_{i k}$ is defined as:

$$
T_{i k} \equiv \rho v_{i} v_{k}+\delta_{i k}\left(\frac{2 \alpha}{5 m} \rho^{5 / 3}\right)-\frac{1}{4}\left(\frac{\hbar}{2 m}\right)^{2} \rho \partial_{i} \partial_{k} \ln (\rho)
$$

(to derive the above equation the following identity has been used: $\left.\rho^{-1} \partial_{i}\left(\rho \partial i \partial_{k} \ln (\rho)\right)=2 \partial_{k}\left(\partial_{i} \partial_{i} \sqrt{\rho} / \sqrt{\rho}\right)\right)$.

Note that although in a superfluid there is no frictional viscosity, nonetheless shear stress may arise from density (pressure) gradients (third term in Eq. (10). As a consequence, vortex formation and drag are possible even with no viscosity.

The force exerted on a condensate by an obstacle moving through it can be calculated from the rate of momentum transfer to the fluid. By integration, one finds the drag force (per unit mass):

$$
F_{k}=\partial_{t} \int_{\Omega} d \Omega J_{k}=-\int_{\Sigma} d \Sigma n_{i} T_{i k}-\int_{\Omega} d \Omega \rho \partial_{k} U
$$

Here $\Sigma$ is the object external surface and $\mathbf{n}$ is a unit vector directed along the outward normal. In the case of an homogeneous flow past an impenetrable object only the first term contributes. In the present case, however, where a partially penetrable object is used (see the following) both contributions are present.

In our calculations we used the full expression (10) for the stress tensor to compute the drag (11). Notice that the quantum term in (10) is expected to be negligible only when $v_{\infty} \gg(\hbar / m R)$, where $R$ is the diameter of the moving object: this is not the case here since these two terms are of comparable magnitude.

\section{METHODS AND CALCULATIONS}

By using a Madelung transformation, equations (11) and (2) can equivalently be written in the form of a time-dependent nonlinear Schrödinger equation (NLSE) 14] involving the complex order parameter $\Psi(\mathbf{r}, t)=$ $\sqrt{n(\mathbf{r}, t)} e^{i \theta(\mathbf{r}, t)}$ :

$$
i \hbar \frac{\partial}{\partial t} \Psi=\hat{H} \Psi
$$

where

$$
\hat{H} \equiv-\frac{\hbar^{2}}{4 m} \nabla^{2}+2 U(\mathbf{r}-\mathbf{V} t)+2 \alpha|\Psi|^{4 / 3}
$$

The link between the two descriptions is provided by the definition

$$
\mathbf{v}(\mathbf{r}, t) \equiv \frac{\hbar}{2 m} \nabla \theta(\mathbf{r}, t)
$$


for the velocity field associated with the phase of the order parameter $\theta(\mathbf{r}, t)$. V in Eq. (13) is the (constant) velocity of the moving object.

By means of a Galilean transformation to the reference frame moving with the object (which will thus appear as stationary in our simulations), the NLSE to be solved becomes:

$$
i \hbar \frac{\partial}{\partial t} \Psi=[\hat{H}+i \hbar \mathbf{V} \cdot \nabla] \Psi
$$

For simplicity, we model the microscopic object inside the moving Fermi gas by means of a repulsive cylindrical "wall" and consider the flow motion perpendicular to it. Due to translational invariance along the axis of the cylinder (which we define as the $z$ axis), the problem thus reduces to finding the density and the velocity of the fluid in the $(x, y)$ plane.

We have numerically solved this 2-D NLSE equation to obtain the long-time dynamics of the fluid due to the motion of the object moving along the $x$ direction. From the time-dependent calculated density $n(x, y, t)$ and velocity $\mathbf{v}(x, y, t)$ we computed the drag acting on the object, according to (11).

Our simulation region is a square cell of side $a=$ $3.2 \mu \mathrm{m}$. We used a uniform mesh to represent the wavefunction $\Psi$, on $512 \times 512$ points in the $(x, y)$ plane. We have used the Runge-Kutta-Gill fourth-order method 21] to propagate in time the solutions of the NLSE. To accurately compute the spatial derivatives appearing in our NLSE, we used a 13-point finite-difference formula [22]. To avoid the outgoing sound waves and/or emitted vortices to interfere with the fluid dynamics after being reflected on on the grid boundaries, we use an exponential absorbing buffer located in the periphery of the cell, as described in Ref. 23. The waves can travel freely in the undamped region (which occupy most of the simulation cell) but are quickly attenuated as they enter the external region, thus preventing unwanted interferences which might spoil the results.

In the following we take $m$ equal to the mass of a ${ }^{6} \mathrm{Li}$ atom. We consider two different density values ("high density" and "low density" in the following) for the UFG, namely $n_{0}=8600 \mu \mathrm{m}^{-3}$ and $n_{0}=880 \mu \mathrm{m}^{-3}$, corresponding to interparticle distances $d \sim 5 \times 10^{-2} \mu \mathrm{m}$ and $d \sim 0.1 \mu m$, respectively.

At equilibrium, the repulsive cylindrical "wall" results in the formation of a circular cavity void of atoms. The object potential $U$ and the associated initial density profile are shown in Fig. (11).

\section{RESULTS AND DISCUSSION}

We made a series of time-dependent calculations, solving Eq. (15) for different values of the speed $V$, and following the dynamics of the systems for several $\mu \mathrm{s}$. We find that there is a critical value $v_{c}$ (which will be quantified in the following) for the object speed $V$ which separates two distinct regimes. When $V<v_{c}$ the fluid profile

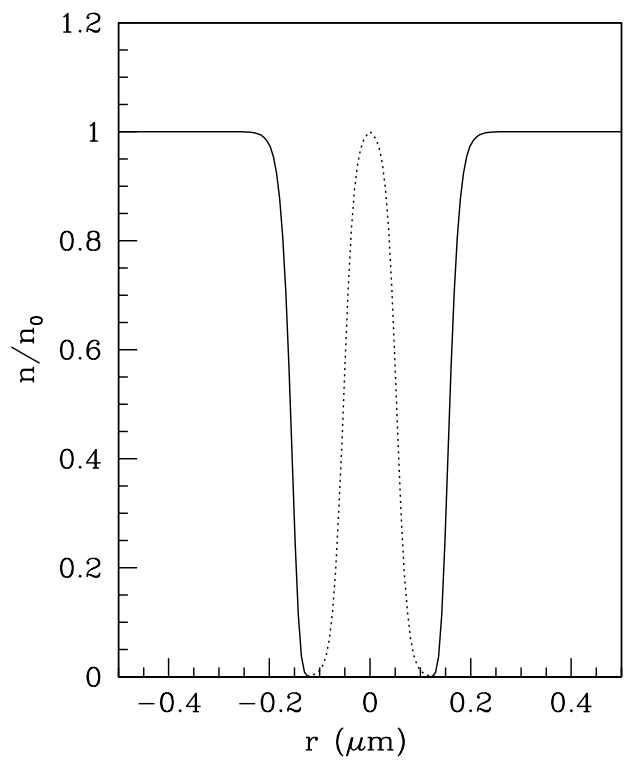

FIG. 1: Equilibrium density profile at $t=0$. The dotted line represents (in arbitrary units) the repulsive potential due to the object.

rapidly evolves with time into a stationary configuration. Both the density and the velocity field for such final configuration are fore-to-aft symmetric. This implies that the drag force on the object is zero, which is another version of the well-known D'Alembert paradox in classical fluids.

In Fig. (2) (lower curve) we show the calculated drag force $F_{x}$ (computed using Eq. (11)) as a function of time when $V<v_{c}$. After a transient where the fluid accomodates under the sudden acceleration of the object, the drag goes eventually to zero when the symmetric (stationary) configuration is reached.

Above $v_{c}$, however, the Fermi gas dynamics changes dramatically: linear (antilinear) vortices are spontaneously created in pairs close to the surface of the object, together with the emission of sound waves.

We show in Fig. (3) a sequence of images taken during the real-time evolution of the system when $V>v_{c}$. The object is moving from left to right. First a localized bow wave moving with supersonic velocity is emitted in front of the cylinder and rapidly moves ahead. This is the result of a "shock" wave produced by the sudden acceleration of the object (we recall that the initial state is with fluid at rest and the object moving at constant velocity).

Then a vortex pair is emitted in the rear, trailing behind (meaning that the pair velocity is lower than that of the moving object). The initial pair is soon overtaken by a pair emitted successively: the vortex lines apparently form a temporarily bound state (but they will eventually split at later times, not shown).

As shown in the upper curve of Fig. (2), in this case 


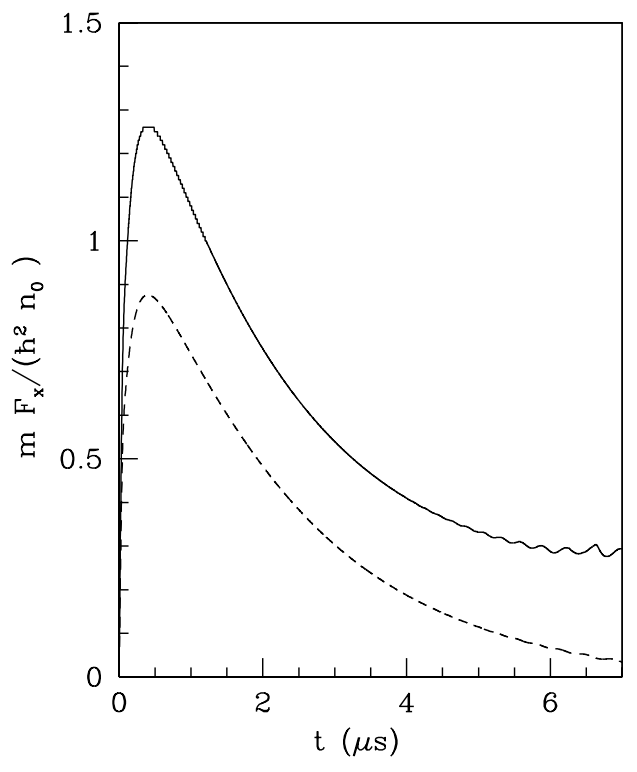

FIG. 2: Drag force during the real-time evolution of the system. Upper curve: $V>v_{c}$, lower curve: $V<v_{c}$.
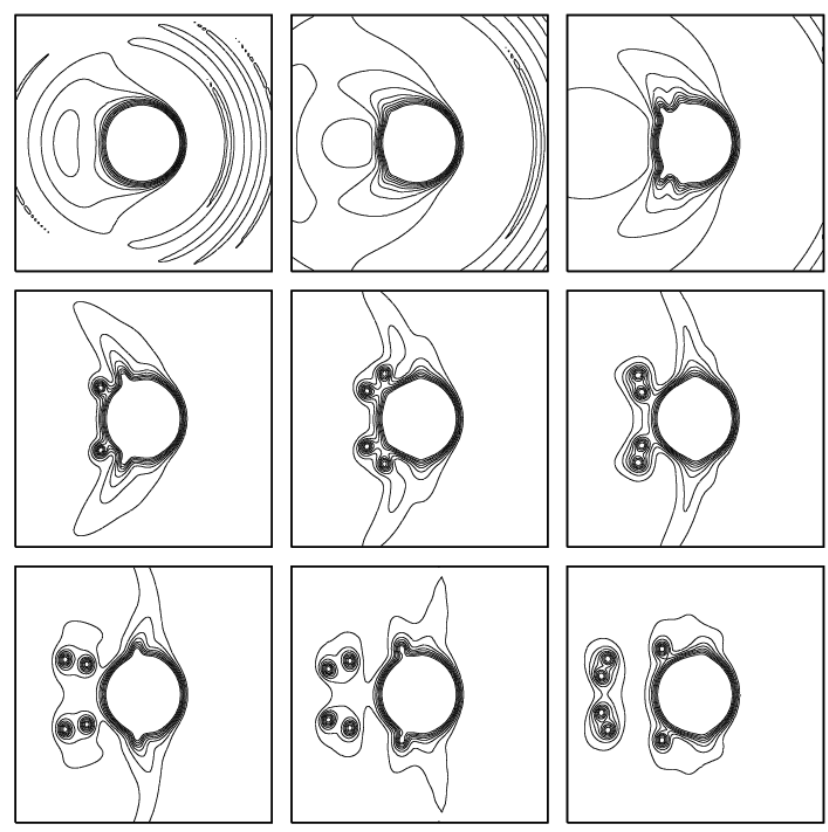

FIG. 3: From left to right, top to bottom: configurations at increasing times for $V / c_{1}=0.76$. Only a portion of the simulation cell is displayed.

the instantaneous calculated drag force relaxes, after a transient, towards a finite nonzero value (with oscillations that reflect the quasi-periodic emission of vortex pairs).

In the simulations of Fig. (3) the velocity $V$ is greater than $v_{c}$, but still below the speed of sound $c_{1}$.

The sequence shown in Fig. (4) is instead obtained for
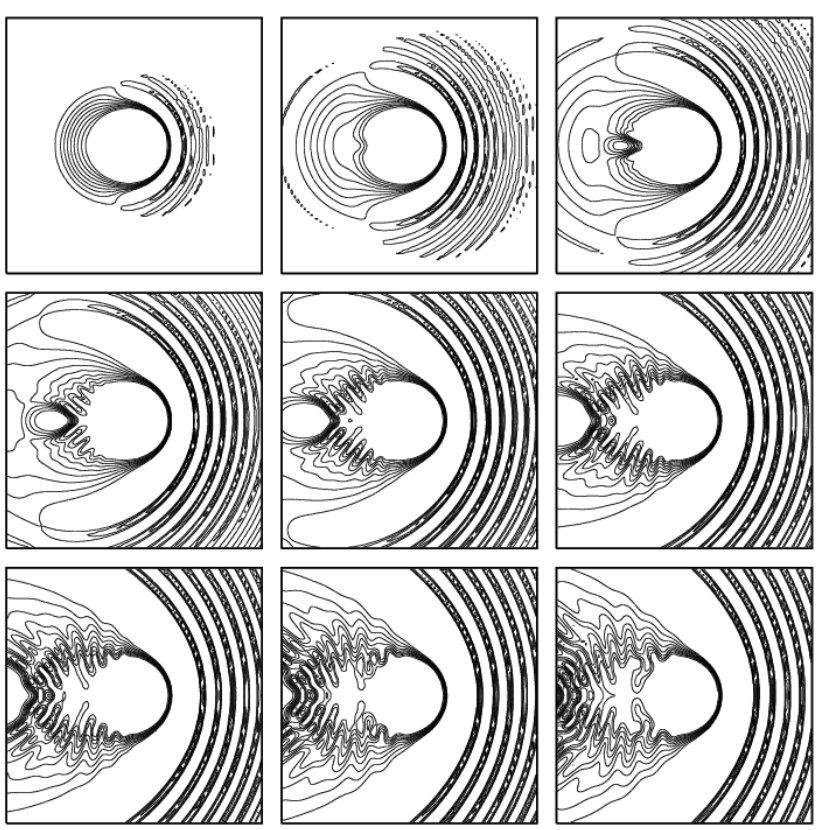

FIG. 4: From left to right, top to bottom: configurations at increasing times for velocity $V / c_{1}=1.44$. Only a portion of the simulation cell is displayed.

a supersonic motion of the object, $V / c_{1}=1.44$. It appears that the vortex shedding frequency is considerably increased with respect to the previous case (similarly to what happens for a BEC, where the shedding frequency is 24] $\propto V^{2}$. Vortex-antivortex pairs are emitted in a semicontinuous way on different part of the rear section of the object, leading to parallel rows of connected vortical lines that eventually decay into separated vortices. Note also the appearance of a rather structured bow sound wave pattern moving along with the object. As time proceeds, the wake region behind the object becomes turbulent due to the superposition of more and more vortices and sound waves.

In order to better characterize the vortex structure, we have selected (from another simulation where $V$ is just above $v_{c}$, so that vortex pairs are emitted with low frequency and thus are well separated from one another) a configuration after a pair of vortex lines have been emitted and moved away from the object, and closely analyzed the vortex structure. We find that the vortex is singly quantized, and that the two vortices of the pair have opposite polarization. The velocity field (not shown) follows very closely the ideal vortex velocity profile, $v(r)=\hbar /(2 m r)$ (here $\left.r=\sqrt{x^{2}+y^{2}}\right)$.

The vortex line structure has an empty core, as shown in Fig. (5), while the core size scale is set by $k_{F}^{-1}$. This is in contrast with calculations based on Bogoliubov-De Gennes calculations [8, 9] where a partially filled core (between 0.2 and $0.3 n_{0}$ ) is predicted due to the presence of some normal liquid coming from pair breaking in the core region, where the velocity is higher. We will discuss in the following the reason for such discrepancy. 


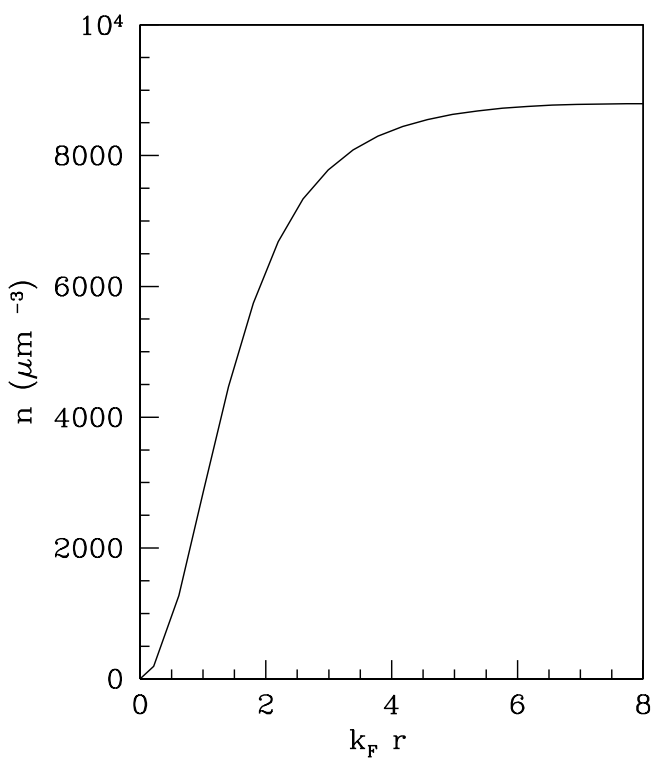

FIG. 5: Density profile in the vortex core region.

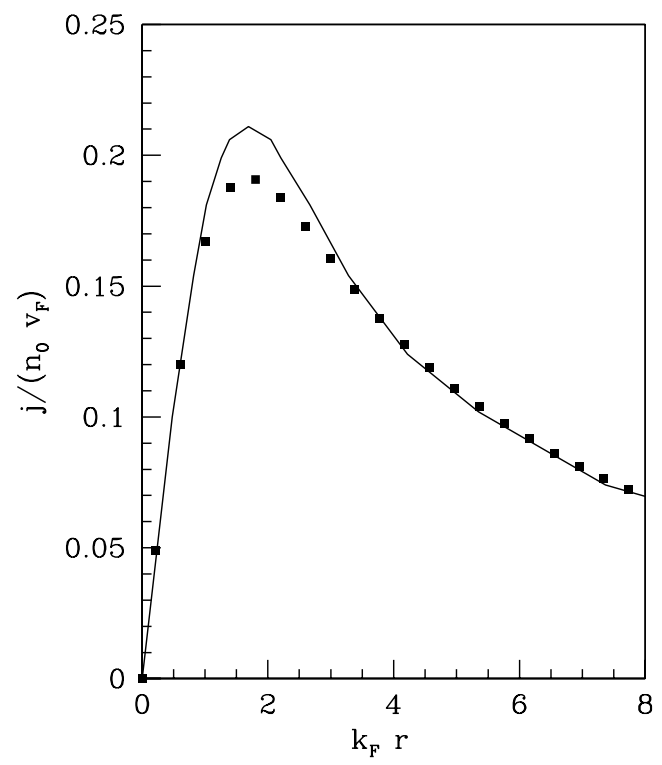

FIG. 6: Current density distribution near the vortex core. Squares: our results. Solid line: BdG calculations from Ref. [9].

We computed the superfluid current density $\mathbf{j}=\rho \mathbf{v}$ circulating around the vortex core (see Fig. (6) ), and found a peak value at $r_{\max }$, in a remarkable overall agreement with the $\mathrm{T}=0$ Bogoliubov-De Gennes calculations of Ref. [9] (shown in Fig. (6) with a solid line).

We find the agreement with the results of Ref. [9] particularly rewarding since it shows that an important superfluid observable related to vorticity can indeed be de- scribed accurately by our Density Functional approach.

The scale of the maximum circulating current is set by the critical velocity which is determined by pair-breaking on the BCS side and by collective excitations on the BEC side [9]. This is why our approach, which cannot obviously describe single-particle processes like pair-breaking, is nonetheless able to reproduce the current pattern. For $r<r_{\max }$ the kinetic energy cost associated with the current flow becomes larger than the condensation energy. Thus $r_{\max }$ gives an estimate of the distance from the core center below which superfluidity is partially suppressed.

We note that the peak position $r_{\max }$ in Fig. (6) coincides with the value of $r$ where the vortex velocity field becomes equal to the local sound velocity, i.e. when

$$
\frac{\hbar}{2 m r} \sim \sqrt{2 \alpha n(r)^{2 / 3} / 3 m}
$$

where $n(r)$ is the vortex core density profile shown in Fig. (5).

In Ref. [16] we have found that, at unitarity, our approach leads to a maximum Josephson current across a barrier which is practically the same as the one obtained from BdG. This suggests that, at unitarity, the current is limited by Landau's criterion for the creation of collective excitations, and not by single particle excitations. It is then not surprising that also in the present case our maximum current is close to that obtained by a much more demanding microscopic calculation based on BdG equations $([8,9])$

It must be said that in Ref. [8, 9] the vortex structure is imposed on the condensate order parameter $\Delta$. Therefore near the core the current goes to zero because $\Delta$ vanishes and the superfluid density vanishes with it, since $\rho_{s} \propto \Delta^{2}$. However in the core region, where the superfluid velocity exceeds the critical value for the creation of single particle excitations, $\rho_{s}<\rho$, so that the total density is non zero even at the core where $\rho_{s}=0$.

On the contrary, in our approach which does not take into account single particle excitations, all the fluid is superfluid, and therefore the vanishing at the core of the superfluid current due to vorticity implies that the total density is zero there.

Results similar to those reported above were obtained from the simulations of the lower density system, the main difference being the shallower vortex cores (which scale as $k_{F}^{-1}$ ). The calculated superfluid current density around the core of an isolated vortex, plotted as a function of $k_{F} r$, is indistinguishable from the one shown in Fig. (6).

We show in Fig. (17) the calculated drag force on the object, as a function of the velocity $V$. Each value is obtained as a time-average [25] of curves like the one shown in Fig. (2) (the average is taken over a time interval where the drag force has already reached a plateau). From these results, a value of the critical velocity $v_{c} \sim 0.4 c_{1}$ is obtained, in agreement with the simple estimate (8). 


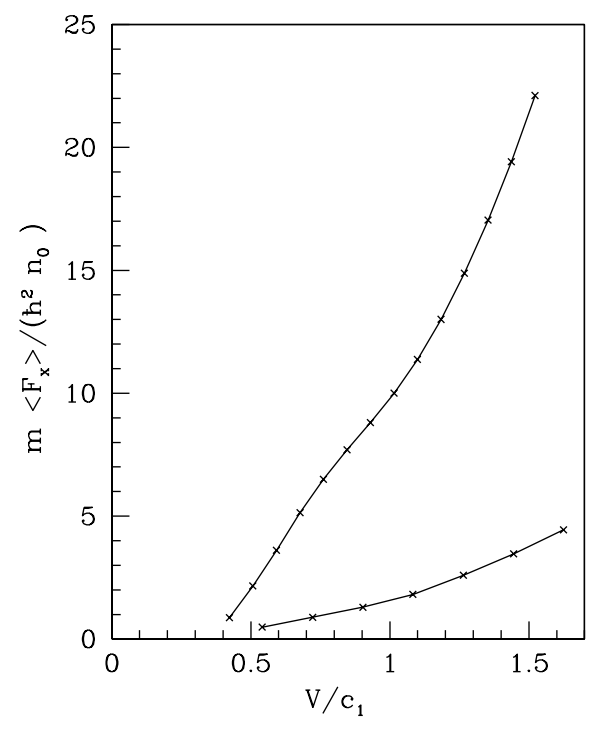

FIG. 7: Average drag force for the high and low density systems, plotted as a function of $V$.

We also find that the drag force first increases linearly with the velocity ("Stoke's law", usually associated with laminar drag) and then turns to a quadratic behavior ("Newton's law", usually associated with turbulent flow), since at supersonic velocities there is also a contribution to the drag associated with sound radiation. A similar behavior is observed in the case of an impenetrable cylinder moving inside a dilute BEC [25].

The phenomenology of the dissipative motion of an object displaced through a UFG, as it appears from our calculations, is qualitatively similar in many aspects, in spite of the different non-linear interactions, to the behavior observed in the case of an object moving in a BEC [20, 25]: the occurrence of vortex emissions in pairs and the associated density patterns are similar in the two systems, and also the behavior of the drag as a function of the object velocity. There are important differences though: the emitted vortices in the UFG are doubly quantized, as expected from fermion pairing; the predicted critical velocity is different; the vortex core structure is also different, scaling in the present case as the inverse Fermi momentum.

\section{CONCLUSIONS}

In conclusion, we have numerically studied the motion of an object in the ultracold unitary Fermi gas. We described the system by using an extended density functional approach, which has been used recently to successfully describe a number of static and dynamical properties of cold Fermi gases.

We find that quantized vortices are spontaneously generated in pairs during the time evolution at supercritical velocities. Moreover, the profile of the current density as a function of the distance from the core is quantitatively close to the one found by the much more demanding solution of the BdG solutions. We explain this agreement by observing that at distances larger than the one corresponding to the maximum current density, in both treatments the superfluid density coincides with the total density, since the speed of the fluid is below its critical value for the creation of single particle excitations. At shorter distances, in the core region, the current density is again similar in the two treatments: in both it vanishes implying that the superfluid density is zero at the center of the vortex. The main difference is that while in our case the superfluid density coincides with the total density, in the $\mathrm{BdG}$ treatment the density may have a contribution from a "normal" component related to single particle excitations and this component provides a nonzero density at the center.

We acknowledge Fondazione CARIPARO (project of excellence "Macroscopic Quantum Properties of Ultracold Atoms under Optical Confinement") and University of Padova (progetto di ateneo "Quantum Information with Ultracold Atoms in Optical Lattices") for partial support.
[1] "The Many-Body X Challenge Problem", formulated by G.F. Bertsch, see R. A. Bishop, Int. J. Mod. Phys. B 15, issue: 10-11, iii (2001).

[2] M.W. Zwierlein et al., Nature (London) 435, 1047 (2005).

[3] S. Gautam, arXiv:1205.5670v1.

[4] E. Lundh and A. Cetoli, Phys. Rev. A 80, 023610 (2009).

[5] A. Bulgac, Y.L. Luo, P. Magierski, K.J. Roche, and Y. $\mathrm{Yu}$, Science 332, 1288 (2011).

[6] M. Rodriguez, G-S. Paraoanu, and P.Torma, Phys. Rev. Lett. 87, 100402 (2001).

[7] G.M. Bruun and L. Viverit, Phys. Rev. A 64, 063606 (2001).

[8] A. Bulgac and Y. Yu, Phys. Rev. Lett. 91, 190404 (2003).
[9] R. Sensarma, M.Randeria and T-L. Ho, Phys. Rev. Lett. 96, 090403 (2006).

[10] A. Bulgac, M. McNeil Forbes, and P. Magierski, in BCSBEC Crossover and the Unitary Fermi Gas, Lecture Notes in Physics, vol. 836, Ed. by W. Zwerger (Springer, Berlin, 2011).

[11] A. Bulgac and Y. Yu, Phys. Rev. Lett. 91, 190404 (2003).

[12] A. Bulgac, Phys. Rev. A 76, 040502(R) (2007).

[13] T. Papenbrock, Phys. Rev. A 72, 041603(R) (2005); A. Bhattacharyya and T. Papenbrock, Phys. Rev. A 74, 041602(R) (2006).

[14] L. Salasnich and F. Toigo, Phys. Rev. A 78, 053626 (2008); L. Salasnich and F. Toigo, Phys. Rev. A 82, 059902(E) (2010). 
[15] S.K. Adhikari and L. Salasnich, Phys. Rev. A 78, 043616 (2008); L. Salasnich, Laser Phys. 19, 642 (2009); S.K. Adhikari and L. Salasnich, New J. Phys. 11, 023011 (2009).

[16] F. Ancilotto, L. Salasnich and F. Toigo, Phys. Rev. A 79, 033627 (2009); L. Salasnich, F.Ancilotto, N. Manini and F.Toigo, Laser Physics19, 636 (2009).

[17] L. Salasnich and F. Toigo, J. Low Temp. Phys. 165, 239 (2011).

[18] F. Ancilotto, L. Salasnich, and F. Toigo, Phys. Rev. A 85, 063612 (2012).

[19] J. Joseph, J. Thomas, M. Kulkarni, and A. Abanov, Phys. Rev. Lett. 106, 150401 (2011).
[20] T. Frisch, Y. Pomeau, and S. Rica, Phys. Rev. Lett. 69, 1644 (1992).

[21] Mathematical Methods for Digital Computers, Ed. by A. Ralston and H. S. Wilf, vol. 1, p. 117 (Wiley, New York, 1960).

[22] M. Pi, F. Ancilotto, E. Lipparini, and R. Mayol, Physica E, 24, 297 (2004).

[23] D. Jin and W. Guo, Phys. Rev. B 82, 094524 (2010).

[24] T. Winiecki, J.F.McCann, and C.S.Adams, Phys. Rev. Lett. 82, 8186 (1999).

[25] T. Winiecki, B. Jackson, J.F. McCann, and C.S.Adams, J. Phys. B: At. Mol. Opt. Phys. 33, 4069 (2000). 